\title{
Expression of aldosterone synthase and adrenocorticotropic hormone receptor in adrenal incidentalomas from normotensive and hypertensive patients: Distinguishing subclinical or atypical primary aldosteronism from adrenal incidentaloma
}

\author{
C.X. $\mathrm{CAO}^{1 *}$, X.C. YANG ${ }^{2}$, Y.X. GAO ${ }^{3}$, M.ZHUANG ${ }^{1}$, K.P. WANG ${ }^{1}$, L.J. $\mathrm{SUN}^{2}$ and X.S. WANG ${ }^{2}$ \\ Departments of ${ }^{1}$ Endocrinology, ${ }^{2}$ Urology and ${ }^{3}$ Nephrology, The Affiliated Hospital \\ of the Medical College of Qingdao University, Shandong, Qingdao 266100, P.R. China
}

Received July 17, 2012; Accepted September 3, 2012

DOI: $10.3892 /$ ijmm.2012.1144

\begin{abstract}
The present study aimed to investigate the expression of aldosterone synthase (CYP11B2), adrenocorticotropic hormone receptor (ACTH-R) and their regulating transcription factors in adrenal incidentalomas (AIs) from normotensive and hypertensive patients to distinguish subclinical or atypical primary aldosteronism (PA) from AIs. Total RNA was extracted from 8 normal adrenal cortices (NAs), 46 AIs, 15 aldosterone-producing adenomas (APAs) and 6 idiopathic hyperaldosteronisms (IHAs). Real-time quantitative polymerase chain reaction (PCR) and immunohistochemistry were performed to determine the mRNA and protein expression of CYP11B2, ACTH-R, steroidogenic factor-1 (SF1) and dosage-sensitive sex reversal, adrenal hypoplasia congenita, critical region on the $\mathrm{X}$ chromosome, gene-1 (DAX-1) in the different tissues. The AI hypertensive subgroup displayed increased plasma aldosterone concentration (PAC) and PAC/PRA ratio (ARR) and decreased plasma renin activity (PRA) compared to the normotensive group. CYP11B2, ACTH-R and SF1 mRNAs were significantly higher in the APA group compared to the other groups, and gradually increased in AI hypertensive samples. DAX-1 mRNA was expressed faintly in PA compared with NA. In normotensiveAI samples, DAX-1 mRNA was higher compared to PA and AI hypertensive samples. Significant differences in gene expression levels in AIs were observed between probable and
\end{abstract}

Correspondence to: Professor L.J. Sun, Department of Urology, The Affiliated Hospital of the Medical College of Qingdao University, No. 59 Haier Road, Shandong, Qingdao 266100, P.R. China

E-mail: yuheangel@yahoo.com.cn

*Contributed equally

Key words: adrenocortical tumour, aldosterone synthase, adrenocorticotropic hormone receptor, steroidogenic factor-1, dosage-sensitive sex reversal, adrenal hypoplasia congenita, critical region on the $\mathrm{X}$ chromosome, gene-1 improbable PA patients. Immunohistochemical results were consistent with those of real-time PCR. Plasma aldosterone levels were positively correlated with CYP11B2, ACTH-R and SF1 mRNA and inversely correlated with DAX-1 mRNA. In conclusion, a significant number of hypertensive-AI patients may have subclinical forms of PA. CYP11B2, ACTH-R and their regulating transcription factors may be noteworthy in distinguishing subclinical PA from AIs.

\section{Introduction}

Due to the increased use of imaging for diagnostic purposes, adrenal incidentalomas (AIs) have become a growing clinical problem. AIs are defined as accidentally discovered, clinically silent adrenal lesions present in abdominal imaging, that was performed for reasons other than the suspected adrenal disease. A number of patients with AIs do not report any clinical problems, but the possibility of a hyperfunctioning tumour or malignancy should be considered. Adrenocortical cancer (ACC) has been reported in 1-9\%, and hormonal hypersecretion in $2-20 \%$ of patients with AIs (1). A hypersecreting tumour is discovered in $2 \%$ of hormonally evaluated patients.

The prevalence of primary aldosteronism (PA) has been increasing in recent years. PA is the most common cause of surgically curable secondary hypertension and affects more than $10 \%$ of the general hypertensive population $(2,3)$. The prevalence of PA is at least $6.8 \%$ in prehypertensive patients, $3.3 \%$ in stage 1 hypertensive patients and $3.1 \%$ in stage 2 hypertensive patients (4).

Accumulating evidence has shown the adverse effect of long-term hyperaldosteronism on cardiovascular morbidity that is independent of blood pressure (BP). However, diagnosing PA may be difficult when clinical and biochemical features vary widely and the criteria for PA are not met, especially in patients with subclinical symptoms, such as stage 1 hypertension, or are found to have atypical laboratory features. When screening patients for PA, plasma renin activity (PRA) determination is sensitive but not specific due to the influence of medications and the high prevalence of low-renin essential hypertension (5). In addition, plasma aldosterone 
concentration/plasma renin activity (PAC/PRA) ratio (ARR) is not reliable in patients with chronic kidney disease (CKD) (6). Thus, the diagnosis of PA remains a challenge for patients with subtle or atypical features.

Increased serum aldosterone within the physiologic range has been observed in normotensive individuals predisposed to the development of sustained hypertension in the future (7). In addition, patients with PA are at a greater risk compared to those with similar BP but without PA of experiencing cardiovascular events and a stroke since long-term hyperaldosteronism leads to vessel and heart damage that is independent of BP (8). Therefore, normalisation of circulating aldosterone is the paramount therapeutic goal for PA (9), and timely identification of subclinical or atypical features of PA is of clinical value.

Aldosterone-producing adenomas (APAs) continue to express high levels of aldosterone synthase (CYP11B2), which maintains the capacity for aldosterone production (10-14). The expression of CYP11B2 appears to be a novel marker for APAs (15). The adrenocorticotropic hormone receptor (ACTH-R, MC2-R) is a member of the 7-transmembrane-domain receptor superfamily. ACTH-R expression is associated with a less aggressive adrenal tumour phenotype and anti-proliferative effects (16). Steroidogenic factor-1 (SF1, NR5A1) and dosage-sensitive sex reversal, adrenal hypoplasia congenita, critical region on the $\mathrm{X}$ chromosome, gene-1 (DAX-1, NR0B1) are 2 orphan nuclear receptors that play major roles in regulating the production of steroidogenic enzymes. SF1 and DAX-1 are functional antagonists of ACTH-R gene transcription in vitro, and DAX-1-induced promoter inhibition requires functional SF1-binding sites within the promoter region of the ACTH-R gene. The functional interplay between DAX-1 and SF1 is complex and includes both agonistic and antagonistic interaction.

The aim of the present study was to investigate the mRNA and protein expression of specific markers of zona glomerulosa, namely CYP11B2 (17), ACTH-R, SF1 and DAX-1, in adrenal tumours. We also aimed to distinguish subclinical or atypical PA from normotensive and mildly hypertensive patients with AIs. Finally, we aimed to determine whether these gene and protein expression levels may act as markers for subclinical PA and contribute to its pathophysiology.

\section{Materials and methods}

Subjects and tissues. Tissues from 8 normal adrenal cortices (NA), 46 non-functional adenomas (AIs), 15 aldosterone-producing adenomas (APAs) and 6 idiopathic hyperaldosteronisms (IHAs) were studied. The AI group was further divided into normotensive (normotensive-AI) and mildly hypertensive (hypertensive-AI) subgroups according to BP (Table I). The clinical and pathological diagnoses were made according to established criteria. Patients with non-functional adrenal adenomas had no signs or symptoms of hormone excess, had normal serum potassium levels and displayed normal suppression of serum cortisol after low-dose dexamethasone treatment. We studied only those patients where the discovery was incidental. In addition, patients with cystic adrenal lesions, hypokalaemia (present or past), high catecholamine levels, abnormal pituitary-adrenal axis function or excess sexual hormones or androgen precursors were not included in the study. APAs were diagnosed on the basis of an elevated plasma aldosterone concentration (PAC) and suppressed plasma renin activity or concentration. The differential diagnosis between APA and hyperaldosteronism due to bilateral idiopathic adrenal hyperplasia was performed using computerised axial tomography, adrenal scintiscan with ${ }^{75} \mathrm{Se}$-methyl-nor-cholesterol after dexamethasone suppression and/or aldosterone/cortisol ratio measurements in adrenal venous blood. All adrenal tumours examined were microscopically confirmed to be adrenocortical adenomas.

Human adrenal glands were obtained from the Affiliated Hospital of the Medical College of Qingdao University. All samples were obtained with full university approval, and written informed consent was obtained from each patient. NA glands were obtained from 8 patients undergoing expanded nephrectomy for kidney cancer. After removing adjacent fat, the tissues were snap-frozen and immediately stored at $-80^{\circ} \mathrm{C}$ until processing.

RNA extraction and real-time RT-PCR. Total RNA was extracted using the Qiagen RNA/DNA kit according to the manufacturer's protocol (Qiagen, Hilden, Germany). The quality and quantity of total RNA were determined using an ND-1000 Spectrophotometer (NanoDrop Technologies, Wilmington, DE, USA). First-strand cDNA was synthesised from $1 \mu \mathrm{g}$ of total RNA using ThermoScript RT (Invitrogen Life Technologies, Carlsbad, CA, USA) and oligo (dT) primers. The primers are listed in the Supplementary material online. For CYP11B2, ACTH-R, SF1 and DAX-1 quantitation, a double-stranded DNA dye, SYBR-Green I (Molecular Probes, Eugene, OR, USA), was used with $9.5 \mu \mathrm{l}$ of SYBR-Green PCR master mix (Applied Biosystems, Foster City, CA, USA) and $100 \mathrm{nmol}$ of each primer. PCR was performed using the ABI 7500 Fast Real-Time PCR System (Applied Biosystems) with a total volume of $20 \mu \mathrm{l} /$ reaction following the reaction parameters recommended by the manufacturer. All real-time PCRs were performed in triplicate. The primers used were as follows: CYP11B2: 5'-CTCTACCCTGTGGGTCTGTTTT-3' (forward), 5'-GGATTATACCGCTCAGGCC-3' (reverse); ACTH-R: 5'-AAGAATAAGAATCTCCAGGCACC-3' (forward), 5'-TCCGCAGCAATCACAGACA-3' (reverse); SF1: 5'-CGCCGCGGGCATGGACTATTCGTA-3' (forward), 5'-CAGGCGCATCCCCACCGTCAGG-3' (reverse); DAX-1: 5'-CTCCGCGCCCTTGCCCAGACC-3' (forward), 5'-GCCGCACGAACAGCCCCAACACT-3' (reverse); and $\beta$-actin: 5'-CAGCACAATGAAGATCAAGATCA-3' (forward), 5'-CGTCATACTCCTGCTTGCTGA-3' (reverse). Real-time quantitative PCR was conducted to analyse the gene expression in different tissues. Gene expression was analysed by relative quantitation with the $2^{-\Delta \Delta \mathrm{Ct}}$ method using $\beta$-actin as an internal control and the NA tissue as calibrator. The results are expressed as the target/internal standard concentration ratio of the sample.

Immunohistochemistry. Immunolocalisation of CYP11B2, ACTH-R, SF1 and DAX-1 was performed using the streptavidin-biotin peroxidase complex method. Briefly, tissue slides were first deparaffinised in xylene, ethanol and water. Then the slides were pretreated in $0.01 \mathrm{M}$ citrate buffer $(\mathrm{pH} 6.0)$ for CYP11B2, ACTH-R, SF1 and DAX-1 and heated in a microwave oven $\left(98^{\circ} \mathrm{C}\right)$ for $5 \mathrm{~min}$. For staining, endogenous 
Table I. Clinical characteristics of the patients with adrenal masses.

\begin{tabular}{|c|c|c|c|c|c|}
\hline & \multirow[b]{2}{*}{$\begin{array}{l}\text { NA } \\
(\mathrm{n}=8)\end{array}$} & \multirow[b]{2}{*}{$\begin{array}{c}\text { APA } \\
(n=15)\end{array}$} & \multirow[b]{2}{*}{$\begin{array}{c}\text { IHA } \\
(\mathrm{n}=6)\end{array}$} & \multicolumn{2}{|c|}{$\mathrm{AI}(\mathrm{n}=72)$} \\
\hline & & & & $\begin{array}{c}\text { Normotensive-AI } \\
\quad(n=40)\end{array}$ & $\begin{array}{l}\text { Hypertensive-AI } \\
\quad(n=32)\end{array}$ \\
\hline Age (years) & $49 \pm 10$ & $47 \pm 13$ & $42 \pm 8$ & $38 \pm 12$ & $41 \pm 9$ \\
\hline Gender $(\mathrm{M} / \mathrm{F})$ & $5 / 3$ & $8 / 7$ & $4 / 2$ & $25 / 15$ & $19 / 13$ \\
\hline BMI $\left(\mathrm{kg} / \mathrm{m}^{2}\right)$ & $22.5 \pm 3.8$ & $27.9 \pm 3.7^{b}$ & $28.5 \pm 6.1^{b}$ & $23.2 \pm 8.6^{\mathrm{d}}$ & $25.4 \pm 7.9^{\mathrm{a}, \mathrm{d}, \mathrm{h}}$ \\
\hline Tumour size (mm) & & $16.8 \pm 12.5$ & $23.6 \pm 10.9^{\mathrm{d}}$ & $34.2 \pm 14.3^{\mathrm{d}, \mathrm{f}}$ & $28.1 \pm 14.6^{\mathrm{d}, \mathrm{e}, \mathrm{h}}$ \\
\hline Morning serum F (nmol/l) & $328.1 \pm 86.9$ & $426.8 \pm 100.4$ & $346.1 \pm 128.0$ & $321.7 \pm 164.8$ & $358.1 \pm 106.4$ \\
\hline ACTH $(\mu \mathrm{g})$ & $17.28 \pm 7.31$ & $22.65 \pm 6.79$ & $26.24 \pm 13.08$ & $24.18 \pm 10.52$ & $21.75 \pm 9.24$ \\
\hline Serum potassium $(\mathrm{mmol} / \mathrm{l})$ & $4.47 \pm 0.58$ & $3.25 \pm 0.98^{b}$ & $3.93 \pm 0.75^{\mathrm{d}}$ & $4.22 \pm 0.87^{\mathrm{d}}$ & $4.06 \pm 0.73^{\mathrm{d}}$ \\
\hline Fasting glucose (mmol/l) & $5.15 \pm 0.54$ & $6.87 \pm 2.30^{\mathrm{b}}$ & $6.96 \pm 1.22^{\mathrm{b}}$ & $5.29 \pm 1.47^{\mathrm{c}}$ & $5.51 \pm 1.50^{c}$ \\
\hline PAC (ng/dl) & & $17.13 \pm 6.17$ & $15.43 \pm 3.50$ & $10.80 \pm 5.10^{\mathrm{d}}$ & $12.70 \pm 5.90^{\mathrm{c}, \mathrm{e}, \mathrm{h}}$ \\
\hline $\operatorname{PRA}(\mu \mathrm{g} / \mathrm{l} / \mathrm{h})$ & & $0.16 \pm 0.07$ & $0.30 \pm 0.15^{\mathrm{c}}$ & $1.37 \pm 0.65^{\mathrm{d}}$ & $0.56 \pm 0.31^{\mathrm{de}, \mathrm{g}}$ \\
\hline ARR & & $87.50 \pm 38.17$ & $62.00 \pm 17.64^{c}$ & $12.60 \pm 10.14^{\mathrm{d}, \mathrm{e}}$ & $23.10 \pm 8.75^{\mathrm{d}, \mathrm{f}, \mathrm{g}}$ \\
\hline Preoperative SBP (mmHg) & $127.6 \pm 16.6$ & $166.9 \pm 28.7^{b}$ & $156.2 \pm 18.0^{\mathrm{b}}$ & $127.3 \pm 13.6^{\mathrm{c}, \mathrm{e}}$ & $150.3 \pm 22.4^{\mathrm{b}, \mathrm{d}, \mathrm{g}}$ \\
\hline Preoperative DBP (mmHg) & $76.5 \pm 12.8$ & $92.8 \pm 13.7^{\mathrm{b}}$ & $91.9 \pm 10.7^{\mathrm{b}}$ & $73.5 \pm 12.8^{\mathrm{d}, \mathrm{f}}$ & $91.3 \pm 17.4^{9}$ \\
\hline Postoperative SBP (mmHg) & $125.5 \pm 16.3$ & $133.8 \pm 13.5$ & $135.5 \pm 14.5$ & $125.5 \pm 16.3$ & $139.5 \pm 15.1^{\mathrm{h}}$ \\
\hline Postoperative DBP (mmHg) & $74.3 \pm 11.2$ & $80.4 \pm 12.0$ & $80.5 \pm 8.9$ & $74.3 \pm 11.2$ & $82.1 \pm 9.0$ \\
\hline
\end{tabular}

Data are the mean $\pm \mathrm{SD} .{ }^{\mathrm{a}} \mathrm{P}<0.05,{ }^{\mathrm{b}} \mathrm{P}<0.01$ compared with $\mathrm{NA} ;{ }^{\mathrm{c}} \mathrm{P}<0.05,{ }^{\mathrm{d}} \mathrm{P}<0.01$ compared with $\mathrm{APA} ;{ }^{\text {e }}<0.05$, ${ }^{\mathrm{P}}<0.01$ compared with IHA; ${ }^{9} \mathrm{P}<0.01,{ }^{h} \mathrm{P}<0.05$ compared with normotensive-AI. BMI, body mass index; ACTH, adrenocorticotropic hormone; PAC, plasma aldosterone concentration; PRA, plasma renin activity; ARR, PAC/PRA ratio; SBP, systolic blood pressure; DBP, diastolic blood pressure; NA, normal adrenal cortex; APA, aldosterone-producing adenoma; IHA, idiopathic hyperaldosteronism; AI, adrenal incidentaloma.

peroxidase activity was blocked by immersing in $3 \% \mathrm{H}_{2} \mathrm{O}_{2}$ in methanol for $10 \mathrm{~min}$ to prevent any nonspecific binding. After blockage with $2 \%$ BSA, the slides were incubated with the primary antibodies against CYP11B2 (dilution 1:300), ACTH-R (dilution 1:100), SF1 (dilution 1:300) and DAX-1 (dilution 1:200) for $90 \mathrm{~min}$ at $37^{\circ} \mathrm{C}$ (Santa Cruz Biotechnology, Inc., Santa Cruz, CA, USA). Each slide was then incubated with the corresponding secondary antibody for $15 \mathrm{~min}$ at $37^{\circ} \mathrm{C}$ and finally incubated with peroxidase-labelled streptavidin (Maixin Biotechnology, China) for $15 \mathrm{~min}$. The reaction products were visualised with diaminobenzidine (Dako, Denmark). All slides were counterstained with haematoxylin. As a negative control, primary antibody was replaced with Tris-buffered saline on sections that were positive for CYP11B2, ACTH-R, SF1 and DAX-1 in preliminary experiments. The tissue sections were examined using an Eclipse E600 microscope equipped with a CCD DXC950 camera.

Statistical analysis. All data are expressed as the means \pm standard error. Data were compared using one-way ANOVA. Spearman's rank correlation was used for correlations. $\mathrm{P}<0.05$ was considered to indicate a statistically significant difference. The Statistical Package for the Social Sciences (SPSS, version 15.0; SPSS, Inc., Chicago, IL, USA) was used for statistical analyses.

\section{Results}

Patient characteristics. The main features of the patients presenting with adrenal tumours are shown in Table I. There were no statistical differences between the 4 groups in terms of age, gender, ACTH level, or cortisol level. Patients with AIs had a larger tumour size compared to APA patients and were thinner than APA and IHA patients. Additionally, hypertensive patients had increased body mass index (BMI) compared with normotensive patients. Systolic and diastolic $\mathrm{BP}$ in AI normotensive patients was lower compared to AI hypertensive and APA patients. As shown in Table I, in APA patients, serum potassium was lower whereas fasting glucose was higher compared to the other groups. While PAC and ARR values were lower in AI patients compared to APA patients, the AI hypertensive subgroup demonstrated increased PAC and ARR and decreased PRA compared with the normotensive patients. A correlation between plasma ARR and age, BMI, BP, plasma potassium or tumour size was not found in any group. PA and AI hypertensive patients demonstrated a significant decrease in systolic and diastolic BP after adrenalectomy.

Expression levels of genes in adrenocortical tumours. The CYP11B2, ACTH-R, SF1 and DAX-1 mRNAs were detected in all human adrenal tissues. The specificity of the amplicons was confirmed by sequencing. Although CYP11B2 mRNA was overexpressed in all samples examined, it was significantly higher in the APA and IHA samples compared with the other groups. The CYP11B2 mRNA had a >2-fold increase in expression in the APA group compared with the NA group. In addition, CYP11B2 mRNA expression was significantly higher in the AI hypertensive samples compared to the normotensive subgroup. ACTH-R and SF1 mRNA expression 

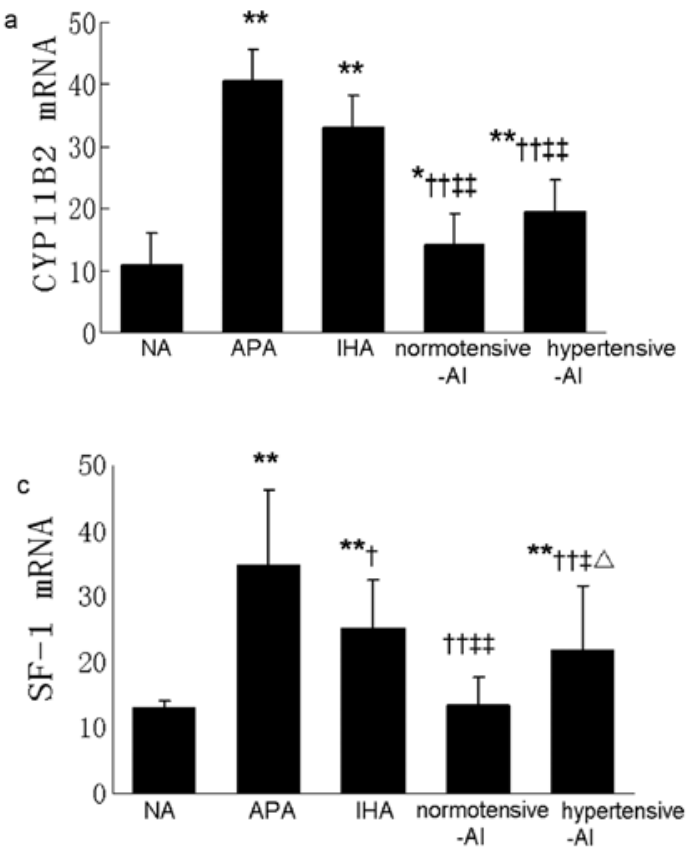
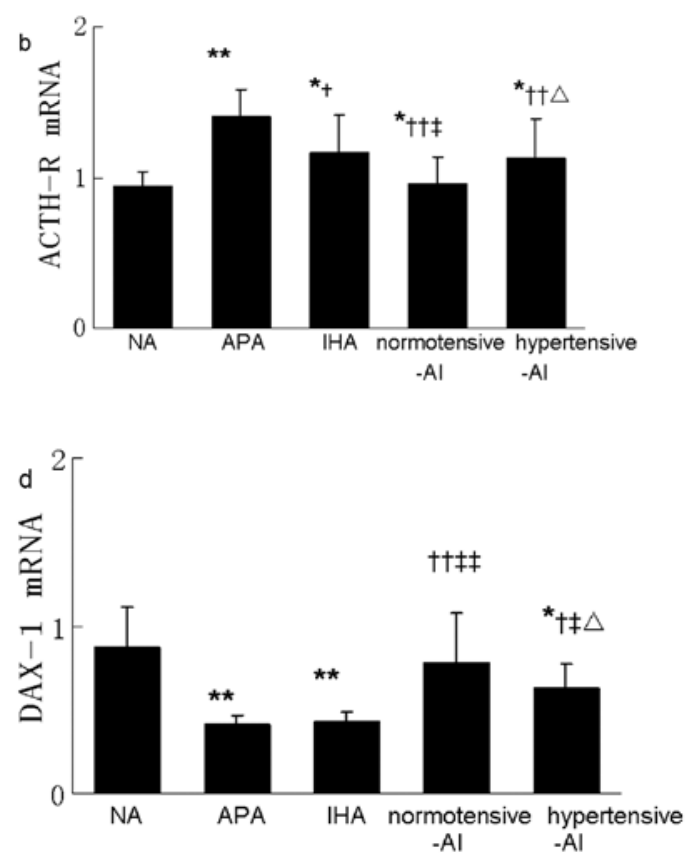

Figure 1. Real-time PCR quantification of (a) CYP11B2, (b) ACTH-R, (c) SF1 and (d) DAX-1 mRNA expression in adrenocortical tumors and NA cortices. NA, normal adrenal cortex; APA, aldosterone-producing adenoma; IHA, idiopathic hyperaldosteronism; AI, adrenal incidentaloma. Expression of the genes was normalised against that of $\beta$-actin, used as an internal control. Data are the means $\pm \mathrm{SD}$ of 3 independent experiments. ${ }^{* *} \mathrm{P}<0.01$, ${ }^{*} \mathrm{P}<0.05$ compared with the control; ${ }^{\dagger} \mathrm{P}<0.05,{ }^{\dagger \dagger} \mathrm{P}<0.01$ compared with APA; ${ }^{\ddagger} \mathrm{P}<0.05,{ }^{\dagger} \mathrm{P}<0.01$ compared with $\mathrm{IHA},{ }^{\Delta} \mathrm{P}<0.05,{ }^{\Delta \Delta} \mathrm{P}<0.01$ compared with normotensive-AI.

Table II. Plasma hormonal data and gene transcription factors of patients with adrenal incidentalomas, with or without probable primary aldosteronism.

\begin{tabular}{lcrr}
\hline & $\begin{array}{c}\text { Probable primary } \\
\text { aldosteronism }(\mathrm{n}=14)\end{array}$ & $\begin{array}{c}\text { Without probable primary } \\
\text { aldosteronism }(\mathrm{n}=58)\end{array}$ & $\begin{array}{c}\text { P-values } \\
\text { PAC }(\mathrm{ng} / \mathrm{dl})\end{array}$ \\
PRA $(\mu \mathrm{g} / \mathrm{l} / \mathrm{h})$ & $13.9 \pm 5.8$ & $10.3 \pm 4.1$ & $<0.05$ \\
ARR & $0.32 \pm 0.20$ & $1.41 \pm 1.21$ & $<0.01$ \\
CYP11B2 & $35.40 \pm 7.64$ & $14.20 \pm 8.75$ & $<0.01$ \\
ACTH-R & $24.08 \pm 6.17$ & $13.34 \pm 9.26$ & $<0.01$ \\
SF1 & $1.23 \pm 0.49$ & $0.92 \pm 0.55$ & $<0.05$ \\
DAX-1 & $25.37 \pm 10.24$ & $0.71 \pm 0.36$ & $<0.01$ \\
\hline
\end{tabular}

The data are the means \pm SD. PAC, plasma aldosterone concentration; PRA, plasma renin activity; ARR, PAC/PRA ratio; CYP11B2, aldosterone synthase; ACTH-R, adrenocorticotropic hormone receptor; SF1, steriodogenic factor-1; DAX-1, dosage-sensitive sex reversal, adrenal hypoplasia congenita, critical region on the $\mathrm{X}$ chromosome, gene- 1 .

was intensely upregulated in APA and slightly upregulated in IHA tissues compared with NA. ACTH-R and SF1 mRNAexpression was also increased in the AI hypertensive compared to the normotensive subgroup. DAX-1 mRNA was underexpressed in the PA group compared with the NA group. In normotensive-AI patients, DAX-1 mRNA expression was also higher compared to PA and AI hypertensive patients. No significant differences in DAX-1 mRNA expression were observed between normotensive-AI and NA samples (Fig. 1).

In APAs, the plasma aldosterone level was positively correlated with CYP11B2 mRNA ( $\mathrm{r}=0.689, \mathrm{P}<0.05)$, ACTH-R mRNA $(r=0.653, P<0.05)$ and $S F 1$ mRNA expression $(r=0.610, P<0.05)$ and inversely correlated with DAX-1
mRNA ( $\mathrm{r}=-1.000, \mathrm{P}=0.000)$. In APAs, the tumour size was negatively correlated with ACTH-R mRNA ( $\mathrm{r}=-0.712$, $\mathrm{P}<0.05)$ and SF1 mRNA expression $(\mathrm{r}=-0.695, \mathrm{P}<0.05)$ but positively correlated with DAX-1 mRNA $(r=0.507, \mathrm{P}<0.05)$. No correlation was observed between the genes and BP before adrenalectomy, nor between the genes and any clinical parameters in the other tissue samples (all $\mathrm{P}>0.05$ ).

A total of 14 subjects $(19.4 \%), 5$ in the normotensive and 9 in the hypertensive subgroup, had an ALD/PRA ratio $>20$ and were regarded as having probable PA. As expected, they had significantly lower PRA and significantly higher plasma ALD and ALD/PRA ratio than the remaining groups $(n=58)$, without differences in age, tumour size or fasting plasma 

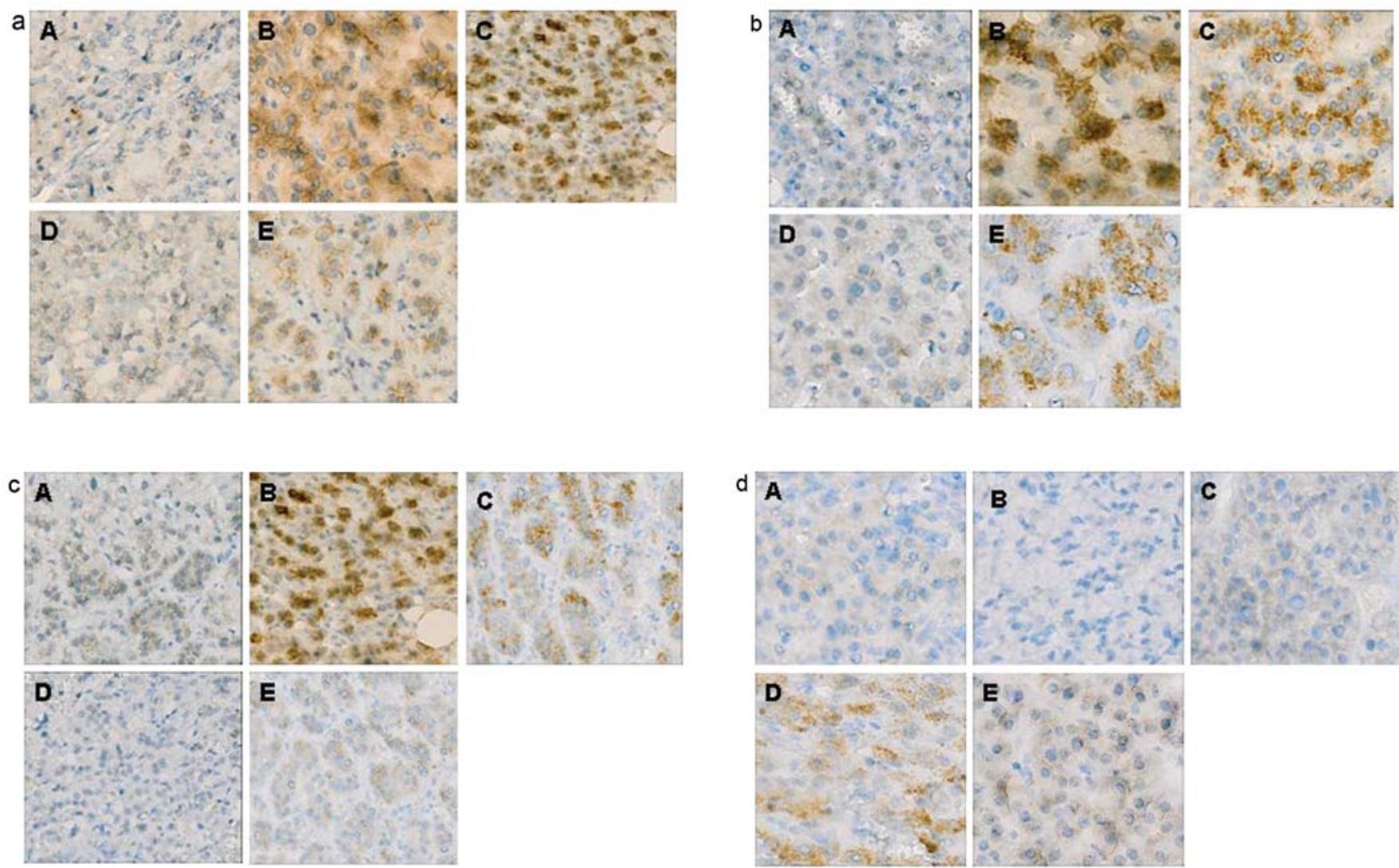

Figure 2. Immunohistochemical analysis of (a) CYP11B2, (b) ACTH-R, (c) SF1 and (d) DAX-1 protein expression in adrenocortical tumours and NA cortex. A, NA cortex; B, APA; C, IHA; D, normotensive-AI; E, hypertensive-AI groups. Magnification, x400. NA, normal adrenal cortex; APA, aldosteroneproducing adenoma; IHA, idiopathic hyperaldosteronism; AI, adrenal incidentaloma.

glucose or potassium. Significant differences in CYP11B2, ACTH-R, SF1 and DAX-1 mRNA levels in AIs were observed between probable and improbable PA patients (all $\mathrm{P}<0.05$ ) (Table II). No correlation was found between the genes and clinical parameters before adrenalectomy (all $\mathrm{P}>0.05$ ).

Immunohistochemical analysis of CYP11B2, ACTH-R, SF1 and DAX-1 in adrenal tumours. Similar to the real-time PCR results, immunohistochemical analysis of CYP11B2, ACTH-R, SF1 and DAX-1 revealed that all four proteins were expressed in all human adrenal tissues sampled. APAs and IHAs displayed strong CYP11B2 and ACTH-R staining in nuclei. Only a weak immunostaining for DAX-1 was occasionally detected. The staining intensity of CYP11B2, ACTH-R and SF1 in AIs was less abundant compared to APAs, while DAX-1 was more abundant in AIs compared to APAs. However, the expression pattern of these proteins was different between the subgroups of AI. The staining of CYP11B2 and ACTH-R was prominent in hypertensive-AI, whereas weak immunostaining for SF1 and DAX-1 was occasionally detected in the hypertensive subgroup (Fig. 2). AI patients with probable PA demonstrated a similar staining with that found in APA patients (data not shown).

\section{Discussion}

In this study, we used real-time RT-PCR and immunohistochemistry analyses to generate expression profiles from
APAs, IHAs, AIs and NA cortex. These profiles were used in part to examine steroidogenic genes and select transcription factors that were differentially expressed among these tissues.

CYP11B2, ACTH-R, SF1 and DAX-1 are often expressed in adrenocortical tumours and cells in NA glands and benign adrenal adenomas are positive for CYP11B2, ACTH-R, SF1 and DAX-1 staining. Accordingly, their important roles in adrenal function and development are well established $(10,18,19)$. The elevated production of aldosterone in APAs is associated with the increased expression of enzymes required for corticosteroid production along with alterations in transcription factors that enhance the expression of steroidmetabolising enzymes. CYP11B2 mRNA reports the most noteworthy change in the APA steroidogenic enzyme gene expression profile. As expected, our results demonstrated that CYP11B2 was upregulated in APAs and IHAs (3- and 2-fold, respectively) compared with NA. A positive correlation between aldosterone secretion and CYP11B2 mRNA was also noted in the APAs. These results are consistent with the increased production of aldosterone that is a characteristic of APAs. Additionally, immunohistochemical analysis of CYP11B2 revealed strong staining in APAs and IHAs. Thus, CYP11B2 may act as a potential pathological marker for both APA and IHA vs non-functional adenomas.

High ACTH-R expression has been observed in aldosteronomas, while a low expression is observed in non-functional adenomas and adrenocortical carcinomas (ACCs) (20), suggesting that aberrant adrenal gene expression and function 
may lead to cell proliferation and the abnormal regulation of steroidogenesis in unilateral adenomas (21). In accordance with previous reports, the results of the present study demonstrated that ACTH-R was overexpressed in APA samples, whereas it was downregulated in IHA, AI and NA samples. In addition, APAs were smaller than IHAs and AIs in this study, which further indicates that increased expression of ACTH-R may inhibit the proliferation of adrenal tumours. SF1 and DAX-1 are functional antagonists of ACTH-R gene transcription, and DAX-1-induced promoter inhibition requires functional SF1-binding sites within the promoter region of the ACTH-R gene (22). SF1 is required for the expression of a variety of steroidogenic enzymes (23). SF1 is amplified and overexpressed in ACC (24) and may be a useful marker in the differential diagnosis of ACC (25). In hyperaldosteronism, $\mathrm{SF} 1$ is important in regulating the endocrine system and is overexpressed in APAs (26). Our results confirm the strong expression of SF1 at the mRNA and protein levels in APAs. Increased SF1 may play an important role in APA formation (27). DAX-1 acts as a general repressor of steroid biosynthesis by inhibiting the transcription of the steroidogenic acute regulatory protein (StAR), 3 $\beta$-hydroxysteroid dehydrogenase and P450scc genes as well as its own transcription $(28,29)$. As our data revealed, DAX-1 displayed faint expression in APAs and IHAs. Increased DAX-1 expression in adrenocortical tumours is associated with a non-functional phenotype, whereas low DAX-1 expression favours mineralocorticoid secretion (30). In addition, aldosterone secretion was positively correlated with ACTH-R and SF1 expression but inversely correlated with DAX-1 expression in APAs. Moreover, tumour size was associated with ACTH-R, SF1 and DAX-1 transcription levels, indicating that these proteins may be involved in APA tumourigenesis. Collectively, these results provide indirect evidence that ACTH-R and its regulating transcription factors, in addition to its known regulatory effects on steroidogenic enzyme gene expression, may also affect the proliferation of adrenal tumours. However, the mechanisms by which these proteins regulate the proliferation of adrenal tumours remain unclear and require further investigation.

Adrenal incidentalomas (AIs) are the most common neoplasia of the adrenal cortex, with a prevalence of up to $6 \%$ in the healthy population (31). Although the vast majority of cases represent endocrine-inactive adrenal adenomas, in individual patients it may prove difficult to distinguish between non-functional and functional adenomas, even with histopathological and immunohistochemical methods. Thus, clinical or molecular markers that would help to distinguish functional adenomas from incidentalomas would be valuable. Our results revealed that AI hypertensive patients had increased PAC and ARR and decreased PRA compared with normotensive patients. After surgery, a significant decrease in systolic and diastolic blood pressure (BP) was observed in hypertensive patients at discharge. These findings indicate that PA is more common in hypertensive patients. Accordingly, approximately $9(28.1 \%)$ patients in the AI hypertensive subgroup were regarded as having probable PA, which was higher than the proportion of normotensive patients (12.5\%). In addition, CYP11B2 expression was significantly higher in the AI hypertensive samples compared to the normotensive subgroup, suggesting APA is more common in hypertensive-
AI patients. It is particularly important to identify APA from AI patients as early as possible since hypertension in APA may be cured by adrenalectomy. Thus, CYP11B2 may be used as a reliable marker for distinguishing APA from AI. Notably, similar expression patterns of ACTH-R and its regulating transcription factors were noted between the AI hypertensive subgroup and APA tissues. Moreover, hypertensive patients had a relatively smaller tumour size compared to normotensive ones. These findings suggest that the overexpression of ACTH-R and SF1, as well as DAX-1 insufficiency, plays a role in both steroid secretory patterns and tumour pathogenesis. By detecting the expression of CYP11B2, ACTH-R, SF1 and DAX-1 in AIs, PA may be identified postoperatively.

Approximately 30 years ago, a 23 -year-old male patient with normotensive PA was reported (32). Recently, Ito et al (4) reported that the prevalence of PA was at least $6.8 \%$ in prehypertensive patients, suggesting a high prevalence of PA among prehypertensive Japanese patients. In accordance with that study, 5 patients in our normotensive had probable PA even though APA is more common in AI patients with hypertension. This means that significant numbers of prehypertensive individuals may have subclinical forms of this disease. We also observed significant differences in CYP11B2, ACTH-R, SF1 and DAX-1 mRNA expression levels between patients with probable PA and those without in AI samples. Immunohistochemical analysis in probable PA patients also demonstrated a similar staining to that in APA. In this regard, CYP11B2, ACTH-R, SF1 and DAX-1 may be used as reliable markers for the early distinction of APA from AI, particularly in patients with normotension. The cause of this normotension remains to be fully elucidated, but the diagnostic criteria of PA should be partly modified.

As we demonstrated in the current study, the plasma aldosterone level was positively correlated with CYP11B2 and ACTH-R mRNA expression and inversely correlated with DAX-1 mRNA in APAs. Our data further indicate that these genes were related to the functional status of APA. However, no significant correlation was found between these genes and the aldosterone/renin ratio or BP before adrenalectomy in AI. The lack of significance of these comparisons may be attributable to the slightly elevated aldosterone and limited number of early-stage PAs.

Collectively, our data suggest that CYP11B2, ACTH-R and their regulating transcription factors may be reliable in distinguishing subclinical PA from AI. It is clinically important to screen for subclinical PA to discover a potentially curable (or improvable) secondary cause of metabolic disorders.

\section{Acknowledgements}

We wish to thank Professor Y.Y. Gao for her worthy technical assistance. This study was supported by grants from the scientific and technological project in Shandong (no. 2011YD18006) and the National Natural Science Foundation of China (no. 81270939).

\section{References}

1. Bülow B, Jansson S, Juhlin C, et al: Adrenal incidentaloma follow-up results from a Swedish prospective study. Eur J Endocrinol 154: 419-423, 2006. 
2. Douma S, Petidis K, Doumas M, et al: Prevalence of primary hyperaldosteronism in resistant hypertension: a retrospective observational study. Lancet 371: 1921-1926, 2008.

3. Monticone S, Viola A, Tizzani D, et al: Primary aldosteronism: who should be screened? Horm Metab Res 44: 163-169, 2012.

4. Ito Y, Takeda R, Karashima S, et al: Prevalence of primary aldosteronism among prehypertensive and stage 1 hypertensive subjects. Hypertens Res 34: 98-102, 2011.

5. Jose A and Kaplan NM: Plasma renin activity in the diagnosis of primary aldosteronism: failure to distinguish primary aldosteronism from essential hypertension. Arch Intern Med 123: 141-146, 1969

6. Plouin PF and Jeunemaitre X: Would wider screening for primary aldosteronism give any health benefits? Eur J Endocrinol 151: 305-308, 2004.

7. Vasan RS, Evans JC, Larson MG, et al: Serum aldosterone and the incidence of hypertension in nonhypertensive persons. $\mathrm{N}$ Engl J Med 351: 33-41, 2004.

8. Mattsson C and Young WF Jr: Primary aldosteronism: diagnostic and treatment strategies. Nat Clin Pract Nephrol 2: 198-208, 2006.

9. Young WF Jr: Minireview: primary aldosteronism - changing concepts in diagnosis and treatment. Endocrinology 144 2208-2213, 2003.

10. Bassett MH, Mayhew B, Rehman K, et al: Expression profiles for steroidogenic enzymes in adrenocortical disease. J Clin Endocrinol Metab 90: 5446-5455, 2005

11. Connell JM, Fraser R, MacKenzie SM, et al: The impact of polymorphisms in the gene encoding aldosterone synthase (CYP11B2) on steroid synthesis and blood pressure regulation. Mol Cell Endocrinol 217: 243-247, 2004.

12. Ye P, Mariniello B, Mantero F, et al: G-protein-coupled receptors in aldosterone-producing adenomas: a potential cause of hyperaldosteronism. J Endocrinol 195: 39-48, 2007.

13. Lenzini L, Seccia TM, Aldighieri E, et al: Heterogeneity of aldosterone-producing adenomas revealed by a whole transcriptome analysis. Hypertension 50: 1106-1113, 2007.

14. Saner-Amigh K, Mayhew BA, Mantero F, et al: Elevated expression of luteinizing hormone receptor in aldosterone-producing adenomas. J Clin Endocrinol Metabol 91: 1136-1142, 2006.

15. Wang T, Satoh F, Morimoto R, et al: Gene expression profiles in aldosterone-producing adenomas and adjacent adrenal glands. Eur J Endocrinol 164: 613-619, 2011.

16. Zwermann O, Schulte DM, Reincke M and Beuschlein F: ACTH 1-24 inhibits proliferation of adrenocortical tumors in vivo. Eur J Endocrinol 153: 435-444, 2005.

17. Boulkroun S, Samson-Couterie B, Golib-Dzib JF, et al: Aldosterone-producing adenoma formation in the adrenal cortex involves expression of stem/progenitor cell markers. Endocrinology 152: 4753-4763, 2011.
18. Shibata H, Ikeda Y, Mukai T, et al: Expression profiles of COUP-TF, DAX-1, and SF-1 in the human adrenal gland and adrenocortical tumors: possible implications in steroidogenesis. Mol Genet Metab 74: 206-216, 2001

19. Val P, Lefrançois-Martinez AM, Veyssière G and Martinez A: SF-1 a key player in the development and differentiation of steroidogenic tissues. Nucl Recept 1: 8, 2003.

20. Reincke M, Beuschlein F, Latronico AC, et al: Expression of adrenocorticotrophic hormone receptor mRNA in human adrenocortical neoplasms: correlation with $\mathrm{P} 450 \mathrm{scc}$ expression. Clin Endocrinol (Oxf) 46: 619-626, 1997.

21. Lacroix A, Bourdeau I, Lampron A, et al: Aberrant G-protein coupled receptor expression in relation to adrenocortical overfunction. Clin Endocrinol (Oxf) 73: 1-15, 2010.

22. Zwermann O, Beuschlein F, Lalli E, et al: Clinical and molecular evidence for DAX-1 inhibition of steroidogenic factor-1dependent ACTH receptor gene expression. Eur J Endocrinol 152: 769-776, 2005.

23. Parker KL and Schimmer BP: Steroidogenic factor 1: a key determinant of endocrine development and function. Endocrine Rev 18: 361-377, 1997.

24. Sasano H, Shizawa S, Suzuki T, et al: Transcription factor adrenal 4 binding protein as a marker of adrenocortical malignancy. Hum Pathol 26: 1154-1156, 1995.

25. Sbiera S, Schmull S, Assie G, et al: High diagnostic and prognostic value of steroidogenic factor-1 expression in adrenal tumors. J Clin Endocrinol Metab 95: E161-E171, 2010.

26. Ouyang J, Hu D, Wang B, et al: Differential effects of downregulated steroidogenic factor-1 on basal and angiotensin II-induced aldosterone secretion. J Endocrinol Invest 34: 671-675, 2011.

27. Hu D, Ouyang J, Wu Z, et al: Elementary studies on elevated steroidogenic factor- 1 expression in aldosterone-producing adenoma. Urol Oncol 30: 457-462, 2012.

28. Zazopoulos E, Lalli E, Stocco DM, et al: DNA binding and transcriptional repression by DAX-1 blocks steroidogenesis. Nature 390: 311-315, 1997.

29. Lalli E, Melner MH, Stocco DM and Sassone-Corsi P. DAX-1 blocks steroid production at multiple levels. Endocrinology 139 4237-4243, 1998

30. Reincke M, Beuschlein F, Lalli E, et al: DAX-1 expression in human adrenocortical neoplasms: implications for steroidogenesis. J Clin Endocrinol Metab 83: 2597-2600, 1998.

31. Reincke M, Beuschlein F, Slawik M and Borm K: Molecular adrenocortical tumourigenesis. Eur J Clin Invest 30 (Suppl 3): S63-S68, 2000.

32. Kono T, Ikeda F, Oseko F, et al: Normotensive primary aldosteronism: report of a case. J Clin Endocrinol Metab 52: 1009-1013, 1981. 